\title{
Treatment outcome of hepatic re-irradiation in patients with hepatocellular carcinoma
}

\author{
Seung Won Seol, MD, MS', Jeong II Yu, MD¹, Hee Chul Park, MD, PhD¹, Do Hoon Lim, MD, PhD', \\ Dongryul Oh, MD', Jae Myoung Noh, MD', Won Kyung Cho, MD', Seung Woon Paik, MD, PhD² \\ Departments of 'Radiation Oncology and ${ }^{2}$ Internal Medicine, Samsung Medical Center, \\ Sungkyunkwan University School of Medicine, Seoul, Korea
}

Purpose: We evaluated the efficacy and toxicity of repeated high dose 3-dimensional conformal radiation therapy (3D-CRT) for patients with unresectable hepatocellular carcinoma.

Materials and Methods: Between 1998 and 2011, 45 patients received hepatic re-irradiation with high dose 3D-CRT in Samsung Medical Center. After excluding two ineligible patients, 43 patients were retrospectively reviewed. RT was delivered with palliative or salvage intent, and equivalent dose of $2 \mathrm{~Gy}$ fractions for $\alpha / \beta=10 \mathrm{~Gy}$ ranged from $31.25 \mathrm{~Gy}_{10}$ to $93.75 \mathrm{~Gy}_{10}$ (median, 44 $\left(\mathrm{Gy}{ }_{10}\right)$. Tumor response and toxicity were evaluated based on the modified Response Evaluation Criteria in Solid Tumors criteria and the Common Terminology Criteria for Adverse Events (CTCAE) ver. 4.0.

Results: The median follow-up duration was 11.2 months (range, 4.1 to 58.3 months). An objective tumor response rate was $62.8 \%$. The tumor response rates were $81.0 \%$ and $45.5 \%$ in patients receiving $\geq 45 \mathrm{~Gy}_{10}$ and $<45 \mathrm{~Gy}_{10}$ respectively $(\mathrm{p}=0.016)$. The median overall survival (OS) of all patients was 11.2 months. The OS was significantly affected by the Child-Pugh class as 14.2 months vs. 6.1 months (Child-Pugh A vs. B, $p<0.001$ ), and modified Union for International Cancer Control (UICC) T stage as 15.6 months vs. 8.3 months (T1-3 vs. T4, $p=0.004)$, respectively. Grade III toxicities were developed in two patients, both of whom received $\geq 50 \mathrm{~Gy}_{10}$.

Conclusion: Hepatic re-irradiation may be an effective and tolerable treatment for patients who are not eligible for further local treatment modalities, especially in patients with Child-Pugh A and T1-3.

Keywords: Hepatocellular carcinoma, Radiotherapy, Toxicity, Re-irradiation

\section{Introduction}

Recent improvements in radiation therapy (RT) techniques as well as knowledge on radiobiological consideration in liver RT have enabled us to deliver enough dose of radiation to get a substantial local control of hepatocellular carcinoma (HCC) [1-6]. Though the scientific evidences of multidisciplinary approach combined with radiation are lacking, RT has been tried frequently as a combined modality for treating naïve HCC patients especially in Asia-Pacific countries [7-10]. RT has also been tried as a salvage treatment in patients with recurrent $\mathrm{HCC}$ which is refractory to or ineligible for other therapies, and showed excellent local control with favorable survival outcomes [11-13].

Received 16 March 2015, Revised 2 November 2015, Accepted 6 December 2015.

Correspondence: Hee Chul Park, MD, PhD, Department of Radiation Oncology, Samsung Medical Center, Sungkyunkwan University School of Medicine, 81 Irwon-ro, Gangnam-gu, Seoul 06351, Korea. Tel: +82-2-3410-2605, Fax: +82-2-3410-2619, E-mail: hee.ro.park@samsung.com

(c) This is an Open Access article distributed under the terms of the Creative Commons Attribution Non-Commercial License (http://creativecommons.org/ licenses/by-nc/4.0/) which permits unrestricted non-commercial use, distribution, and reproduction in any medium, provided the original work is properly cited.

www.e-roj.org 
In the clinical reality, we face the situations that re-irradiation is needed for the patients with recurrent HCC, mainly due to the lack of available local salvage modalities for the very patients [14]. The biological properties of liver regeneration as a parallel organ have been studied primarily in surgical settings following partial hepatectomy, but several studies also showed unirradiated liver regions regenerating after proton radiation and stereotactic body RT [15-17]. Additionally, there have been recent experimental animal studies demonstrating the regeneration kinetics of hepatic proliferation after liver irradiation $[18,19]$. Hepatic re-irradiation in patients with HCC are being applied based on such theoretical basis and increasing needs in the clinic, but studies on re-irradiation have been seldom reported.

In this study, we evaluated the efficacy and toxicity of repeated high-dose 3-dimensional conformal RT (3D-CRT) for patients with recurrent $\mathrm{HCC}$, and also investigated the factors affecting the treatment outcomes.

\section{Materials and Methods}

This study was approved and exempted from the permission requirement by the Institutional Review Board of Samsung Medical Center (IRB No. 2013-09-078).

\section{Patients}

From 1998 to 2011, 45 patients with unresectable HCC received liver directed second RT with high dose 3D-CRT in Samsung Medical Center. The eligibility criteria for repeated RT were as follows: 1) HCC not eligible for or refractory to other therapies; 2) persistent disease after initial RT. We included 43 patients by excluding two ineligible patients, one with immediate followup loss and the other who underwent liver transplantation after second RT. Radiation-related and medical records of the eligible patients were retrospectively reviewed.

\section{Radiation therapy}

Computed tomography (CT) scans for RT planning were done with each patient in a supine position, with both arms raised above the head. CT simulation data were transferred to a 3D-CRT planning system (from 1998 to 2003, PROWESS, Alliant Medical Technology, Chico, CA, USA; from 2004 to 2011, PINNACLE, The Philips Medical System, Madison, WI, USA). The tumor, normal liver, kidneys, spinal cord, and bowels were contoured and reconstructed to form a 3-demensional representation.

The clinical target volume (CTV) was regarded same as the gross tumor volume. To determine the cranial-caudal margins, the diaphragmatic excursion during respiration was visualized using fluoroscope, and CTV was expanded by the amplitude of this excursion (1.5-2.5 cm). RT was delivered with a 6- to 15MV linear accelerator. Treatment planning for re-irradiation was performed with an emphasis on maximizing nonirradiated liver volume and minimizing the overlapped volume of irradiation in the skin using 3D-CRT technique. Total dose was designed under tentative guidelines so that the normal liver volume irradiated with over half of the prescribed dose should not exceed $50 \%$ of the total liver volume. The total dose was determined by considering the doses of the remnant functioning liver, gastro-duodenum, and large bowel by the discretion of the treating physician considering previous radiation dose to the critical structures, interval from the previous course of radiotherapy. The re-irradiation dose ranged from 30 to 55 Gy (median, 45 Gy) in daily 2 to 15 Gy (median, 3 Gy) fractions.

\section{Follow-up}

Tumor response was evaluated on serial CT scans 4 to 6 weeks after completion of second RT and then at 3 months intervals. Tumor response was determined using the criteria from the modified Response Evaluation Criteria in Solid Tumors for HCC [20] based on the reports of the diagnostic radiologists. Survival was calculated from the date that second RT has started.

\section{Evaluation of radiation toxicity}

Physical examinations and chemistry profiles including liver function were checked on every follow-up visit. Toxicities were assessed using the Common Terminology Criteria for Adverse Events (CTCAE) ver. 4.0. Radiation-induced liver disease (RILD) was separated into 'classic' and 'non-classic' RILD. Classic RILD was characterized by the presence of anicteric ascites and the elevation of alkaline phosphatase levels to at least a 2-fold increase over pretreatment values in the absence of tumor progression. Non-classic RILD was defined as the elevation of liver transaminases more than five times the upper limit of normal or CTCAE grade 4 levels in patients with baseline values more than five times the upper limit of normal within 3 months after completion of RT, or a decline in liver function (measured by worsening of the Child-Pugh score by 2 or more) $[21,22]$.

\section{Statistics}

Tumor response according to RT dose was compared using 
the chi-square test. Survival was estimated using the KaplanMeier method and compared using the log-rank test to determine potential prognosticators. The Cox proportional hazard regression analysis was used for multivariate analysis. A $p$-value $<0.05$ was considered to be statistically significant. All aforementioned analyses were performed using IBM SPSS Statistics ver. 20 (IBM, Armonk, NY, USA).

\section{Results}

\section{Patient characteristics and treatment}

Patient characteristics at the time of second RT are summarized in Table 1. The median age of all patients was 59 years (range, 29 to 71 years). Modified Union for International Cancer Control (UICC) T stage was determined based on the viable tumor evaluated before starting the second $\mathrm{RT}$, and the group of 43 patients was comprised of 30 (69.8\%) T1-T3 and 13 (30.2\%) T4 patients. Vascular invasion was accompanied in 24 patients (55.8\%) and 33 patients (76.7\%) had multiple tumors. Majority of patients had a clinical liver function of Child-Pugh

Table 1. Patient characteristics

\begin{tabular}{lc}
\hline Characteristics & No. of patients (\%) \\
\hline Sex & \\
Male & $35(81.4)$ \\
Female & $8(18.6)$ \\
Age (yr) & $24(55.8)$ \\
$<60$ & $19(44.2)$ \\
$\geq 60$ & \\
Child-Pugh class & $36(83.7)$ \\
A & $7(16.3)$ \\
B & \\
Modified UICC T stage & $3(7.0)$ \\
T1 & $11(25.6)$ \\
T2 & $16(37.2)$ \\
T3 & $13(30.2)$ \\
T4 & $19(44.2)$ \\
Vascular invasion & $24(55.8)$ \\
No & \\
Yes & $10(23.3)$ \\
Multiplicity & $33(76.7)$ \\
Single & \\
Multiple & $27(62.8)$ \\
Initial AFP (ng/mL) & $16(37.2)$ \\
$<400$ & $11(25.6)$ \\
$\geq 400$ & $32(74.4)$ \\
Liver cirrhosis & No \\
Yes &
\end{tabular}

UICC, Union for International Cancer Control; AFP, alpha-fetoprotein.
A classification (83.7\%).

Median interval between first and second RT was 13.8 months (range, 1.0 to 58.1 months). Because different doses per fraction were used, the equivalent doses to 2 Gy fractions $\left(E Q D_{2 G y}\right)$ for $\alpha / \beta$ ratio of 10 were calculated. The dose of first $R T$ ranged from $32.5 \mathrm{~Gy}_{10}$ to $93.8 \mathrm{~Gy}_{10}$ (median, $48.8 \mathrm{~Gy}_{10}$ ). Second RT was delivered with palliative $(n=24)$ or salvage $(n=19)$ intent, and the RT dose ranged from $31.25 \mathrm{~Gy}_{10}$ to $93.75 \mathrm{~Gy}_{10}$ (median, $44 \mathrm{~Gy}_{10}$ ).

Target volumes of second RT in the liver were overlapped with the first RT site in 13 patients (30.2\%), adjacent with the volume of first RT in 21 patients (48.8\%), and apart from the first RT site in 9 patients (20.9\%). Cumulative RT dose in the overlapping target volume from two RT sessions ranged from 65.0 $\mathrm{Gy}_{10}$ to $117.0 \mathrm{~Gy}_{10}$ (median, 90.6 $\mathrm{Gy}_{10}$ ). Gastro-duodenum was re-exposed in 16 patients (37.2\%).

During the follow-up after second RT, additional transarterial chemoembolization (TACE) was performed in 11 patients, radiofrequency ablation in one patient, third $R T$ in three patients, and fourth RT in one patient.

\section{Radiographic in-field tumor response rates}

The radiographic in-field tumor responses were evaluable in all patients. An objective tumor response rate was $62.8 \%$ with complete response in 8 lesions (18.6\%) and partial response in 19 (44.2\%). The tumor response rates were $81.0 \%$ and $45.5 \%$ in patients receiving $\geq 45 \mathrm{~Gy}_{10}$ and $<45 \mathrm{~Gy}_{10}$, respectively $(\mathrm{p}=$ 0.016 ) (Table 2). Local control rate was $84.0 \%$ at 1 -year and 77.6\% at 2-year (Fig. 1A).

\section{Survival analysis and predictors of survival}

The median follow-up duration calculated from the initiation of the second RT was 11.2 months (range, 4.1 to 58.3 months). During the follow-up period, eight patients remained alive and 35 died. The overall survival (OS) rates at 1- and 2-year were $57 \%$ and 38\%, respectively (Fig. 1B). The median OS was 11.2

Table 2. Relationship between the radiation dose and response

\begin{tabular}{cccc}
\hline \multirow{2}{*}{ Response } & \multicolumn{2}{c}{ RT dose $\left(E^{2} D_{10 / 2}\right)^{\text {a) }}$} & p-value \\
\cline { 2 - 3 } & $\geq 45 \mathrm{~Gy}_{10 / 2}$ & $<45 \mathrm{~Gy}_{10 / 2}$ & \\
\hline CR/PR & $17(81.0)$ & $10(45.5)$ & 0.016 \\
SD/PD & $4(19.0)$ & $12(55.5)$ & - \\
\hline
\end{tabular}

Values are presented as number (\%).

$R T$, radiation therapy; $E Q D$, equivalent dose; $C R$, complete response; $P R$, partial response; $S D$, stable disease; $P D$, progressive disease. a) EQD in 2 Gy fractions for $\alpha / \beta=10 \mathrm{~Gy}$. 
A

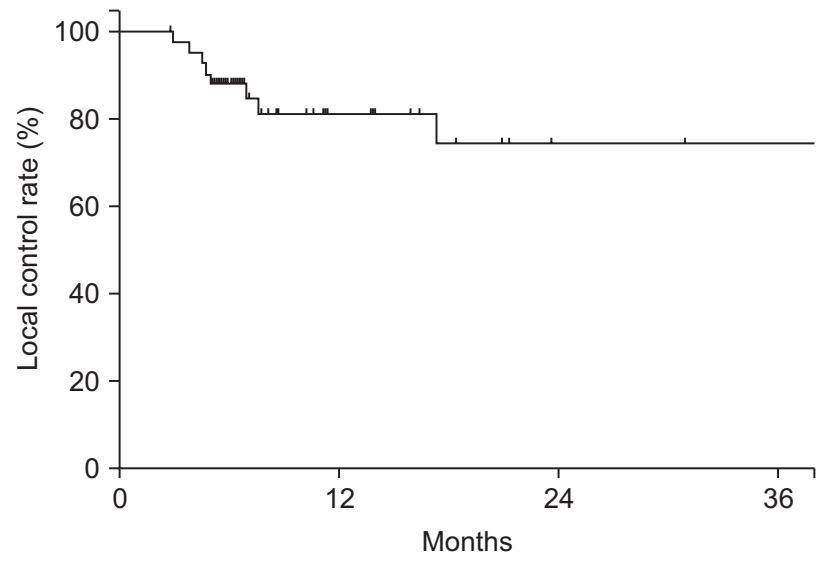

B

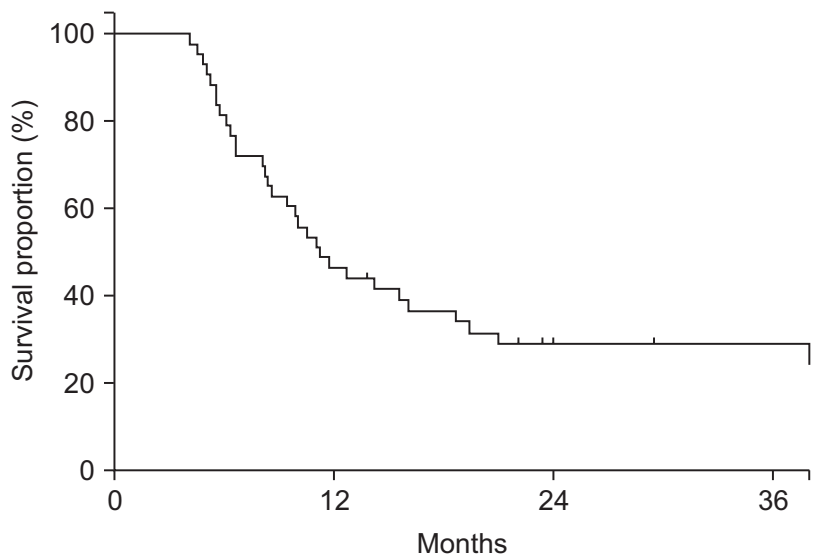

Fig. 1. (A) In-field local control and (B) overall survival of all patients.

A

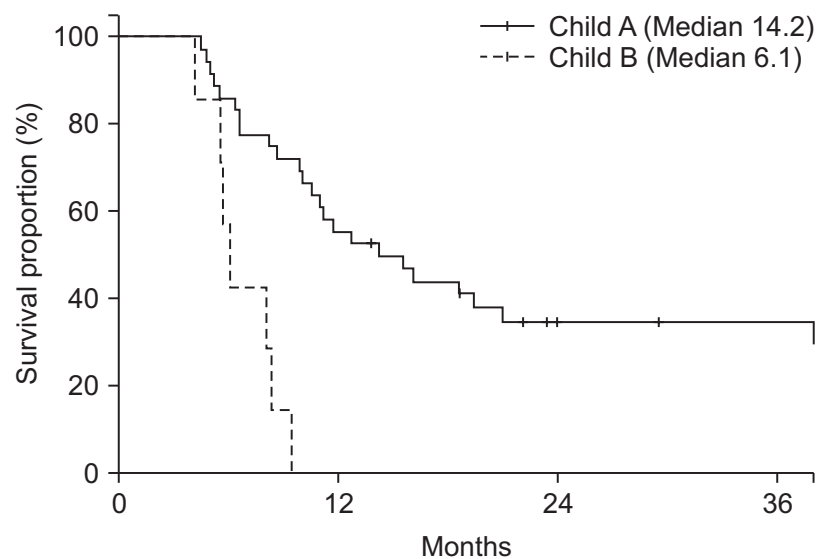

C

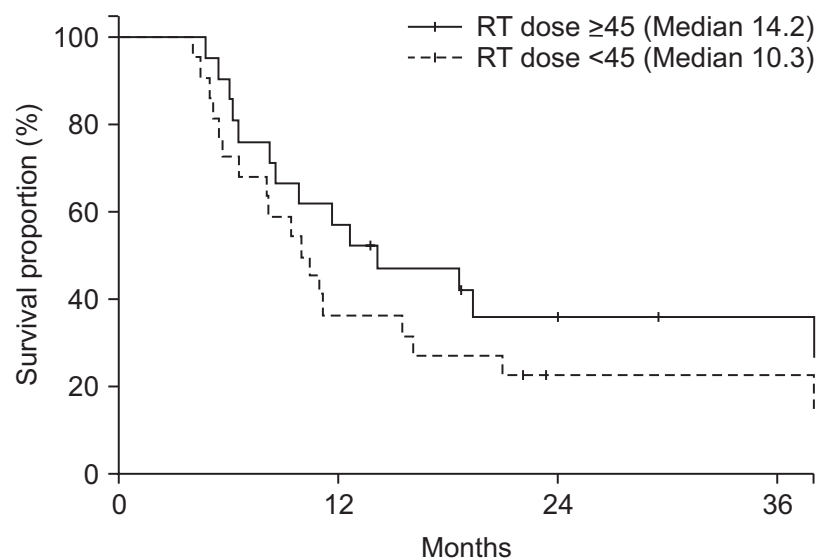

B

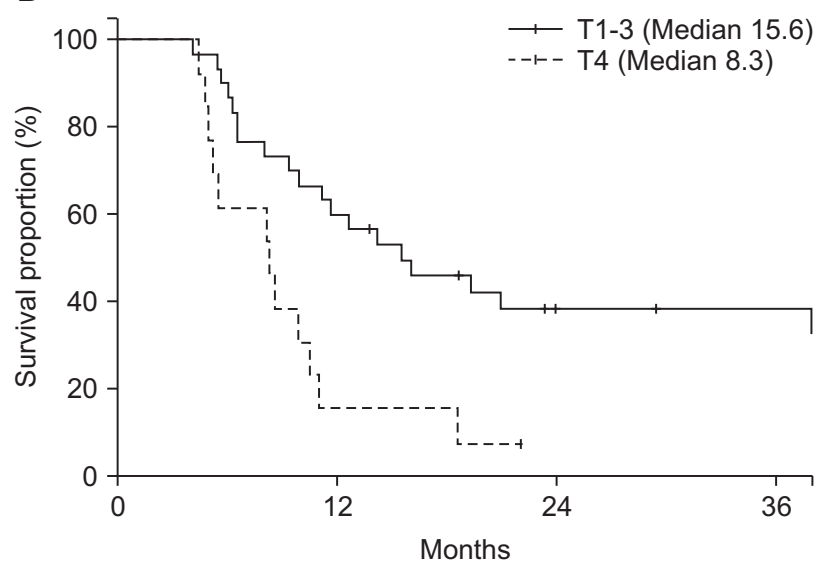

D

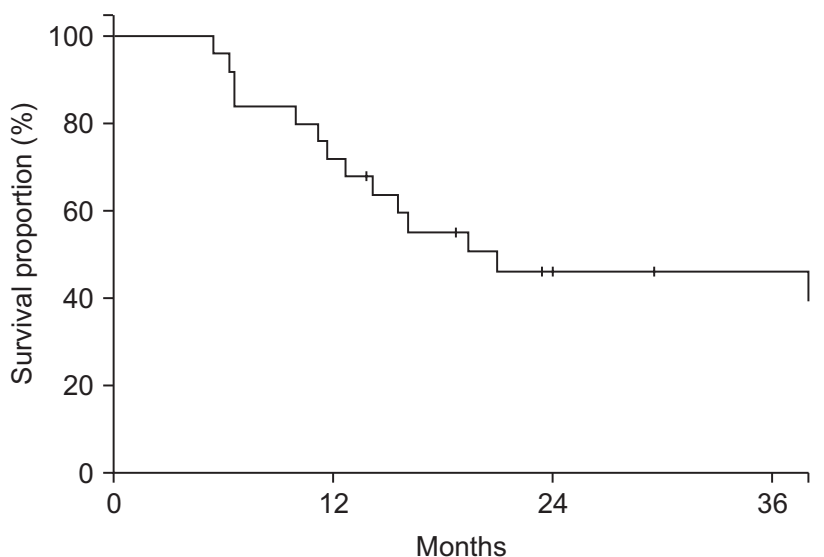

Fig. 2. Overall survival according to (A) Child-Pugh class, (B) the modified Union for International Cancer Control (UICC) T stage, (C) radiation therapy (RT) dose, and (D) in selected clinical cases (Child-Pugh A, the modified UICC T stage T1-3).

months, and it was significantly affected by the Child-Pugh class and the modified UICC T stage. The median OS were 14.2 months vs. 6.1 months (Child-Pugh A vs. B, $p<0.001$ ) (Fig. 2A) and 15.6 months vs. 8.3 months (T1-3 vs. T4, $p=0.004$ ) (Fig. $2 \mathrm{~B})$, respectively (Table 3). Higher RT dose ( $\left.\geq 45 \mathrm{~Gy}_{10}\right)$ tended to be associated with better OS (14.2 months vs. 10.3 months, 
Table 3. Univariate analysis of prognostic factors for overall survival (OS)

\begin{tabular}{|c|c|c|c|}
\hline Variable & No. & Median OS (mo) & $p$-value \\
\hline Age (yr) & & & 0.753 \\
\hline$<60$ & 24 & 11.7 & \\
\hline$\geq 60$ & 19 & 9.9 & \\
\hline Sex & & & 0.595 \\
\hline Male & 35 & 11.2 & \\
\hline Female & 8 & 8.6 & \\
\hline Child-Pugh class & & & $<0.001$ \\
\hline$A$ & 36 & 14.2 & \\
\hline B & 7 & 6.1 & \\
\hline Modified UICC T stage & & & 0.004 \\
\hline $1-3$ & 30 & 15.6 & \\
\hline 4 & 13 & 8.3 & \\
\hline Vascular invasion & & & 0.015 \\
\hline No & 19 & 39.2 & \\
\hline Yes & 24 & 11.2 & \\
\hline Extrahepatic disease & & & 0.537 \\
\hline No & 35 & 11.7 & \\
\hline Yes & 8 & 8.6 & \\
\hline Treatment aim & & & 0.010 \\
\hline Salvage & 19 & 21.0 & \\
\hline Palliative & 24 & 9.4 & \\
\hline Radiation dose $\left(\mathrm{Gy}_{10 / 2}\right)$ & & & 0.199 \\
\hline$<45$ & 22 & 10.3 & \\
\hline$\geq 45$ & 21 & 14.2 & \\
\hline Response & & & 0.816 \\
\hline $\mathrm{CR} / \mathrm{PR}$ & 27 & 19.4 & \\
\hline $\mathrm{SD} / \mathrm{PD}$ & 16 & 13.4 & \\
\hline AFP (ng/mL) & & & 0.726 \\
\hline$<400$ & 27 & 12.7 & \\
\hline$\geq 400$ & 16 & 8.6 & \\
\hline
\end{tabular}

UICC, Union for International Cancer Control; CR, complete response; $P R$, partial response; $S D$, stable disease; $P D$, progressive disease; AFP, alpha-fetoprotein.

$p=0.199$ ) (Fig. 2C). Median OS increased to 24 months when patients with poor prognostic factor (Child-Pugh Class B and modified UICC T-stage T4) were excluded (Fig. 2D). Overlap of RT volumes in the liver or gastro-duodenum between first and second RT was not significantly related to OS.

By multivariate analysis (Table 4), the Child-Pugh class and the modified UICC T stage were significant prognostic factors affecting $0 S$ ( $p=0.001$ and $p=0.009$, respectively).

\section{Treatment-related toxicities}

The profiles of treatment-related RT toxicity are displayed in Table 5. Overall CTCAE grade 1 or 2 toxicities were developed in 18 patients (41.9\%). Nausea, anorexia, and gastrointestinal pain were the most common toxicities, but the acute
Table 4. Multivariate analysis of prognostic factors for overall survival

\begin{tabular}{lccc}
\hline \multicolumn{1}{c}{ Factor } & Hazard ratio & $\begin{array}{c}95 \% \text { Confidence } \\
\text { interval }\end{array}$ & p-value \\
\hline Age & 1.118 & $0.511-2.446$ & 0.780 \\
Sex & 1.065 & $0.394-2.878$ & 0.901 \\
Child-Pugh class & 5.555 & $2.015-15.317$ & 0.001 \\
Modified UICC T stage & 2.782 & $1.293-5.984$ & 0.009 \\
Radiation dose & 0.688 & $0.335-1.413$ & 0.309 \\
\hline
\end{tabular}

UICC, Union for International Cancer Control.

Table 5. The profiles of treatment-related toxicity after hepatic re-irradiation

\begin{tabular}{lcccc}
\hline \multirow{2}{*}{ Adverse event } & \multicolumn{5}{c}{ No. of patients with CTCAE grade (\%) } \\
\cline { 2 - 5 } & $1-2$ & 3 & 4 & 5 \\
\hline Anorexia & $4(9.3)$ & - & - & - \\
Nausea & $6(14.0)$ & - & - & - \\
Dysphagia & $2(4.7)$ & - & - & - \\
Diarrhea & $1(2.3)$ & - & - & - \\
Gastrointestinal pain & $6(14.0)$ & - & - & - \\
Duodenal ulcer & - & $1(2.3)$ & - & - \\
Cough & $1(2.3)$ & - & - & - \\
Pneumonitis & - & $1(2.3)$ & - & - \\
\hline
\end{tabular}

CTCAE, Common Terminology Criteria for Adverse Events.

symptoms were resolved spontaneously or with supportive care. Two patients developed grade 3 or higher toxicity during follow-up period, both of them received $\geq 50 \mathrm{~Gy}_{10}$ at the time of second RT. One patient suffered from grade 3 duodenal perforation and the other from grade 3 pneumonitis. The patient with grade 3 duodenal perforation underwent three times of RT before the adverse event with 45 Gy in 15 fractions and 11 months after the first course with $54 \mathrm{~Gy}$ in 18 fractions followed by $48 \mathrm{~Gy}$ in 16 fractions after 6 months after the second course. The site of perforation seemed to correlate with the site repetitively exposed with 3 times of RT. Although it was difficult to differentiate RILD from deteriorating liver function associated with disease progression or the side effect of TACE, no definitive classic or non-classic RILD was observed during the follow-up period.

\section{Discussion and Conclusion}

In currently available management guidelines for HCC, individual modalities such as sorafenib or TACE are suggested as a sole treatment for unresectable HCC [23]. However, in practice, the orchestration of multimodality treatments is 
needed for successful treatment of individual patients [8]. Usually the HCC management guidelines suggest initial therapy only at diagnosis. However, since the progression after initial therapy is also an important issue, appropriate salvage treatments should also be recommended [8]. Due to the successful combined treatments for unresectable HCC patients, more patients are getting to outlive the duration of expected benefits from initial palliative RT, and require second session of liver-directed RT for intrahepatic recurrence which is refractory to or ineligible for other loco-regional therapies.

In the liver-directed re-irradiation, special cautions are required as HCC patients tend to have poorer liver function and cirrhosis after repeated liver-directed therapies $[4,6]$. Furthermore, re-irradiation to the close proximity with adjacent radiosensitive organs such as stomach and duodenum might cause serious treatment toxicity and its resultant treatment-related death $[5,24,25]$. Hepatic re-irradiation in patients with HCC has not been studied with an exception of proton re-irradiation [14]. The present study evaluates efficacy, toxicity, and the factors which affect survival rate of hepatic re-irradiation in patients with unresectable HCC.

We compared the treatment outcomes of patients who received $\geq 45 \mathrm{~Gy}_{10}$ to that of patients who received $<45 \mathrm{~Gy}_{10}$ because the tolerance radiation dose of gastrointestinal tract is generally considered to be approximately $45 \mathrm{~Gy}_{10}$ [26]. In the current study, the dose-response relationship existed with statistical significance. The tumor response rates were $81.0 \%$ and $45.5 \%$ in patients receiving $\geq 45 \mathrm{~Gy}_{10}$ and $<45$ $\mathrm{Gy}_{10}$, respectively. It suggests that dose-response relationship in HCC identified by former studies may also be applied to re-irradiation situation [27-29]. Survival showed positive relationship with $\mathrm{RT}$ dose as well, but with no statistical significance. The group with high dose RT ( $\geq 45 \mathrm{~Gy}_{10}$ ) showed higher median OS of 14.2 months compared to 10.3 months for low dose RT. Statistical significance might be achieved in the future trials using larger sample size.

There were two prognostic factors predicting survival in our analysis: Child-Pugh class and the modified UICC T-stage. While the median OS was 11.2 months, the results showed that it was significantly affected by these two factors. The median OS increased to 24 months when the patients with both ChildPugh Class B and modified UICC T-stage T4 were excluded (Fig. 2D). This survival figure is close to the formerly reported median OS of first RT as a combined modality on HCC patients $[7,9,10,29,30]$, and it could indicate that re-irradiation can be effective in selected group of patients with Child-Pugh A and T1-3 stage. The other potential prognosticators need further investigation through future studies.

In the toxicity analysis, which is one of most important concerns in re-irradiation situation, two patients developed grade 3 or higher toxicity during follow-up period, both of them received $\geq 50 \mathrm{~Gy}_{10}$ at the time of second RT. Special caution is required in cases of second RT dose is higher than $50 \mathrm{~Gy}_{10}$ to preserve adjacent gastro-duodenum and reduce the risk of radiation pneumonitis. Although the overlapping of the target volume in the liver or gastro-duodenum did not influence the survival statistics, the grade 3 duodenal perforation developed in a patient who received three times of radiation exposure to the gastro-duodenum. Therefore, specific caution is required in the re-exposure of gastro-duodenum and further studies are needed to reveal the relationship between the dose volumetric parameters after re-irradiation and the development of serious complications.

On the other hand, low complication rate (2 out of 43 patients) may imply that re-irradiation is feasible for selected HCC patients. Treatment result in the current study suggests that re-irradiation can be delivered safely with careful consideration to adjacent organs, even when the treatment field of second RT overlaps with the first RT field in the liver or gastro-duodenum. Considering the current development of RT techniques in precision and accuracy, re-irradiation is expected to be applied more frequently as an option for salvage or palliation.

Limitations of this study include retrospective approach, heterogeneous characteristics of patient group, small sample size and not being able to utilize composite plans to evaluate dose-volume histogram parameters and toxicity. The findings of this study regarding the efficacy and low complication rate of re-irradiation on HCC patients can provide useful data and essential perspective for future prospective studies, but the result is not yet conclusive. It can only suggest a tentative guideline for hepatic re-irradiation and should be further improved by prospective studies with large sample size to establish treatment guidelines and dose constraints for these treatments.

In conclusion, the hepatic re-irradiation for HCC patients may be feasible and tolerable option. It was effective in selected group of patients with Child-Pugh A classification and modified UICC T1-3 stage, and the toxicities were manageable except in two patients who had grade 3 complications. However, to define the role and indication of hepatic reirradiation in HCC patients, further prospective studies with larger study population are warranted. 


\section{Conflict of Interest}

No potential conflict of interest relevant to this article was reported.

\section{Acknowledgments}

This research was partly supported by Basic Science Research Program through the National Research foundation of Korea (NRF) funded by the Ministry of Education, Science and Technology (NRF-2012R1A1A2042414). This study was partly supported by Samsung Medical Center grant (GF01130081).

\section{References}

1. Dawson LA. Overview: where does radiation therapy fit in the spectrum of liver cancer local-regional therapies? Semin Radiat Oncol 2011;21:241-6.

2. Dawson LA, Ten Haken RK. Partial volume tolerance of the liver to radiation. Semin Radiat Oncol 2005;15:279-83.

3. Kim B, Park HC, Oh D, et al. Development of the DVH management software for the biologically-guided evaluation of radiotherapy plan. Radiat Oncol J 2012;30:43-8.

4. Kim YI, Park HC, Lim DH, et al. Changes of the liver volume and the Child-Pugh score after high dose hypofractionated radiotherapy in patients with small hepatocellular carcinoma. Radiat Oncol J 2012;30:189-96.

5. Yoon $H_{1} \mathrm{Oh} \mathrm{D}$, Park $\mathrm{HC}$, et al. Predictive factors for gastroduodenal toxicity based on endoscopy following radiotherapy in patients with hepatocellular carcinoma. Strahlenther Onkol 2013;189:5416.

6. Yu JI, Park HC, Lim DH, Park WY. Predictive factors for ChildPugh score elevation in hepatocellular carcinoma patients treated with conformal radiation therapy: dose-volume histogram analysis. Tumori 2013;99:164-71.

7. Oh D, Lim DH, Park HC, et al. Early three-dimensional conformal radiotherapy for patients with unresectable hepatocellular carcinoma after incomplete transcatheter arterial chemoembolization: a prospective evaluation of efficacy and toxicity. Am J Clin Oncol 2010;33:370-5.

8. Park HC, Seong J, Tanaka M, et al. Multidisciplinary management of nonresectable hepatocellular carcinoma. Oncology 2011;81 Suppl 1:134-40.

9. Yoon SM, Lim YS, Won HJ, et al. Radiotherapy plus transarterial chemoembolization for hepatocellular carcinoma invading the portal vein: long-term patient outcomes. Int J Radiat Oncol Biol Phys 2012:82:2004-11.

10. Yu Jl, Park HC, Lim DH, et al. Scheduled interval trans-catheter arterial chemoembolization followed by radiation therapy in patients with unresectable hepatocellular carcinoma. J Korean Med Sci 2012;27:736-43.

11. Bae SH, Park HC, Lim DH, et al. Salvage treatment with hypofractionated radiotherapy in patients with recurrent small hepatocellular carcinoma. Int J Radiat Oncol Biol Phys 2012;82:e603-7.

12. Lim $\mathrm{DH}$, Lee $H$, Park $H C$, et al. The efficacy of high-dose 3-dimensional conformal radiation therapy in patients with small hepatocellular carcinoma not eligible for other local modalities. Am J Clin Oncol 2013;36:162-6.

13. Park JH, Yoon SM, Lim YS, et al. Two-week schedule of hypofractionated radiotherapy as a local salvage treatment for small hepatocellular carcinoma. J Gastroenterol Hepatol 2013:28:1638-42.

14. Hashimoto T, Tokuuye K, Fukumitsu N, et al. Repeated proton beam therapy for hepatocellular carcinoma. Int J Radiat Oncol Biol Phys 2006;65:196-202.

15. Farach A, Quesada J, Teh BS. Liver regeneration following repeat SBRT. J Gastrointest Oncol 2015;6:E2-6.

16. Ohara $\mathrm{K}$, Okumura $T$, Tsuji $H$, et al. Radiation tolerance of cirrhotic livers in relation to the preserved functional capacity: analysis of patients with hepatocellular carcinoma treated by focused proton beam radiotherapy. Int J Radiat Oncol Biol Phys 1997;38:367-72.

17. Stinauer MA, Diot $\mathrm{Q}$, Westerly DC, Schefter TE, Kavanagh BD. Fluorodeoxyglucose positron emission tomography response and normal tissue regeneration after stereotactic body radiotherapy to liver metastases. Int J Radiat Oncol Biol Phys 2012;83:e613-8.

18. Gu K, Lai ST, Ma NY, et al. Hepatic regeneration after sublethal partial liver irradiation in cirrhotic rats. J Radiat Res 2011;52: 582-91.

19. Zhao JD, Jiang GL, Hu WG, Xu ZY, Wang CF. Hepatocyte regeneration after partial liver irradiation in rats. Exp Toxicol Pathol 2009:61:511-8.

20. Lencioni R, Llovet JM. Modified RECIST (mRECIST) assessment for hepatocellular carcinoma. Semin Liver Dis 2010;30:52-60.

21. Pan $C C$, Kavanagh $B D$, Dawson $L A$, et al. Radiation-associated liver injury. Int J Radiat Oncol Biol Phys 2010;76(3 Suppl):S94-100.

22. Xu ZY, Liang SX, Zhu J, et al. Prediction of radiation-induced liver disease by Lyman normal-tissue complication probability model in three-dimensional conformal radiation therapy for primary liver carcinoma. Int J Radiat Oncol Biol Phys 2006;65: 189-95.

23. European Association For The Study Of The Liver; European Organisation For Research And Treatment Of Cancer. EASLEORTC clinical practice guidelines: management of hepatocellular carcinoma. J Hepatol 2012;56:908-43.

24. Chen LS, Lin HC, Hwang SJ, Lee FY, Hou MC, Lee SD. Prevalence of gastric ulcer in cirrhotic patients and its relation to portal 
hypertension. J Gastroenterol Hepatol 1996;11:59-64.

25. Kim H, Lim DH, Paik SW, et al. Predictive factors of gastroduodenal toxicity in cirrhotic patients after three-dimensional conformal radiotherapy for hepatocellular carcinoma. Radiother Oncol 2009;93:302-6.

26. Marks LB, Yorke ED, Jackson A, et al. Use of normal tissue complication probability models in the clinic. Int J Radiat Oncol Biol Phys 2010;76(3 Suppl):S10-9.

27. Liang SX, Zhu XD, Lu HJ, et al. Hypofractionated threedimensional conformal radiation therapy for primary liver carcinoma. Cancer 2005;103:2181-8.
28. Park HC, Seong J, Han KH, Chon CY, Moon YM, Suh CO. Doseresponse relationship in local radiotherapy for hepatocellular carcinoma. Int J Radiat Oncol Biol Phys 2002;54:150-5.

29. Park W, Lim DH, Paik SW, et al. Local radiotherapy for patients with unresectable hepatocellular carcinoma. Int J Radiat Oncol Biol Phys 2005;61:1143-50.

30. Yu Jl, Park HC, Lim DH, et al. Prognostic index for portal vein tumor thrombosis in patients with hepatocellular carcinoma treated with radiation therapy. J Korean Med Sci 2011;26: 1014-22. 